\title{
Survey on Cloud-Based Video Suggestions with Categorization of Users in Shared Systems
}

\author{
Muthu Pradeepa S P \\ UG Student, Department of Computer Science and \\ Engineering, S.A.Engineering College, \\ Poonamallee- Avadi Road, Veeraraghavapuram, \\ Thiruverkadu Post, Chennai - 600077

\section{Sangeetha $S$} \\ UG Student, Department of Computer Science \\ and Engineering, S.A.Engineering College, \\ Poonamallee- - Avadi Road, Veeraraghavapuram, \\ Thiruverkadu Post, \\ Chennai - 600077
}

\author{
Priya $\mathbf{R}$ \\ UG Student, Department of Computer Science \\ and Engineering, S.A.Engineering College, \\ Poonamallee - Avadi Road, \\ Veeraraghavapuram, Thiruverkadu Post, \\ Chennai - 600077 \\ Prabhu S \\ Associate Professor, Department of Computer \\ Science and Engineering, S.A. Engineering \\ College, Poonamallee - Avadi Road, \\ Veeraraghavapuram, Thiruverkadu Post, \\ Chennai - 600077
}

\begin{abstract}
With the rapid growth in multimedia services and the enormous offers of video contents in online social networks, users have difficulty in obtaining their interests. Therefore, various personalized recommendation systems have been proposed. In addition, none of them has considered both the privacy of users' contexts (e,g., social status, ages and hobbies) and video service vendors' repositories, which are extremely sensitive and of significant commercial value. To handle these problems, it's been proposed a cloud-assisted differentially private video recommendation system based on distributed online learning.In our project we proposed the new optimization technique for recommendation. The video recommendation is based on user's behavior (user's interest) and also using the pattern mining for video tag search recommendation. We have search option as sub category search and global search in our application. Facing massive multimedia services and contents in the Internet is based the content provider. In that group of providers we need to find out the irrelevant content promoters. Content promoters are usually trying to promote their contents to social media service or video service sites in internet. In our project Based on the user's interest we can detect and avoid the irrelevant content and content promoters.
\end{abstract}

KEYWORDS- Video recommendation, Cloud sharing, User based recommendation, Categorized recommendation.

\section{INTRODUCTION}

Online video distribution systems, out of which YouTube is the majority trendy, provide features that allow users to post a video as a response to a discussion topic. These features open opportunities for users to introduce polluted content,or just contamination, keen on the scheme. So we locate For example, spammers might position an unrelated video as response to a popular one, aiming at increasing the likelihood of the response being viewed by a larger number of users.

Personalization makes it quick and easy to dynamically find a video based on a user's preference in the number of video, and enables easy access. For this personalization a recommendation system is necessary

In the suggest PDPR scheme personalization technique collect and analyzes the performance pattern, such as: the target user's performance pattern for contents, statistical information for the overall user's performance patterns, a user's personal summary or partiality information from side to side the examination of a user's computing environment, a communication service, and the preferred device types such as a mobile phone, personal computer, etc.

A content-based recommendations scheme recommend the mainly possible coordinated item, then compares the recommendation list to a user's previous input data or compared to preference items. A content-based recommendations scheme is based on in sequence pointed and usually uses a rating method which is used in the information searching. The rating method calculates a user's partiality in sequence and items in a recommendation list. It recommends the mainly possible plan in a user's profile. This method has the advantage with easily adopt in recommendation result and allow additional rapidly recommendation. But it has problems with difference result and efficient refer to appropriate rating configuration.

\subsection{Cloud Computing}

1. Cloud computing is the release of computing and storage space ability as a examine to a mixed community of endrecipients.

2. Cloud computing is a universal term for anything that involves transport hosted services over the Internet.

3. A model for transport in sequence knowledge services in which resources are retrieved from the internet through web-based tools and applications.

4. Cloud computing is so surname since the in sequence living being accessed is establish in the "clouds", and does not require a user to be in a specific place to gain access to it.

5. Cloud computing refers to request and services accessible more than the Internet.

6. These services are accessible from information center all in excess of the world, which together are referred to as the "cloud."

7. The idea of the "cloud" simplifies the many network connections and computer system concerned in online services.

8. Cloud computing is computing reproduction, not a technology.

9. In this representation of computing, all the servers, networks, applications and other basics associated to data centers are made accessible to IT and end users. 
10. Cloud computing is a kind of computing that is equivalent to grid computing.

11. It relies on allocation computing capital rather than having local servers or personal devices to handle applications.

12. Software as a service-SaaS has develop into a ordinary relief representation for a lot of production applications, together with accounting, collaboration, customer relationship management (CRM), organization information systems

13. Platform as a service -It is a group of cloud computing services that give a computing stage and a key stack as a service. Along with SaaS and IaaS, it is a service representation of cloud computing.

14. Infrastructure as a service - IaaS refers not to a engine that does all the work, but only to a ability given to business that offers users the control of extra storage space in servers and data centers.

\subsection{Features of cloud computing:}

- Consumption based billing - Pay per use seems to be the charming characteristic for cloud.

- Rapid Elasticity - clients can enlarge or reduce the capacity within a substance of minutes. This can be by addition example in cases of EC2 or by just rising memory in some additional cases.

- Self Service Based Model - user contain the skill to upload, construct, organize, list, direct, and report on their industry services on require.

- Location and Device Independence-Users can right to use the server from end to end the internet from any place and from any device including the I Phone and Androids.

- Multi-tenancy-There is no require for extra resources similar to actual estate, electricity etc for the servers.

- Reliability-consistency is better by having many sites for the same service, such that if single faces an outage, the other can take over the consignment for the time being.

- Ease of Maintenance-Since, the application don't need to be install on every person's processor, protection of such a centralized application becomes much easier.

- Reduce Financial Overheads-By utilize cloud computing, you could reduce financial expenses which are associated to possessing huge IT departments and your system could do away with better portions of the process of IT.

- Less Prone to Failure-Also, with no massive records stored upon your individual systems, they'll be more useful and less prone to failure whether it is temporary or else.

- Safety- With each one of these basics, security must be a concern. And password safety.

\section{RELATED WORKS}

Ming Cheung,James She, and Zhanming Jie in 2015. A media immense data structure that uses this watched ponder is proposed as another alternative to customer made marks and social outlines for disciple/adherent recommendation and sex recognizing confirmation. To the best of our understanding, this is the essential attempt in this field to show and plan such a wonder for mass customer conferred pictures along to more helpful desire methods. Right when customer social outlines are quite recently open to prohibitive get-togethers, these customer shared pictures are ended up being a less difficult and convincing differentiating choice to discover customer affiliations. This exertion consider more than 360000 customer open portrayals from two social course of action of affiliations, Sky rock and 163 Weibo, in which 3 million supporter/followed associations are incorporated. It is watched that the common analogies from customers with an aficionado/followed connection exhibit moderately unrivaled similarities.
Arjmand Samuel, Muhammad I. Sarfraz, Saleh Basalamah, Arif Ghafoor in 2015. Proposes a blend approach where assurance necessities are gotten in a get the opportunity to control system and present a structure for association and usage of security plans. The focus is to allow a customer, not a system or security executive to make strife free methodologies for their online sight and sound data. An additional need is that such a course of action be setting careful. The development of intuitive media enormous data for dispersing and sharing of massive measures of information raises imperative security and assurance concerns. One such concern is the plan and prerequisite of security techniques remembering the true objective to securely direct access of sight and sound huge data. A couple of researchers have pointed out that for honest to goodness approval of insurance procedures, the security necessities should be gotten in get the opportunity to control systems.

Marko Tkalcic, Andrej Košir, Jurij Tasic in 2011. To exhibit a uniting structure that positions the examination work, that has been done thusly far scattered, in a three stage appear. We give instances of research that cover distinctive parts of the acknowledgment of sentiments and the fuse of emotions into recommender structures. Recommender frameworks have generally depended on information driven descriptors for substance and client displaying. As of late we have seen an expanding number of endeavors to utilize feelings in die rent approaches to enhance the nature of recommender frameworks. A key issue in recommender systems, especially concerning customer driven structures, is to move from data driven assessment criteria to customer centered examination criteria. We have not tended to this issue in this paper as it appears to greater estimations. In this paper we present a bringing together structure that positions the exploration work, that has been done as such far in a scattered way, in a three phase demonstrate. We give cases of research that cover different parts of the discovery of feelings and the consideration of feelings into recommender frameworks.

Shuhui Jiang, Xueming Qian, Jialie Shen, Yun Fu, Tao Mei in 2015. A maker topic indicates based shared isolating (ATCF) methodology is proposed to support careful reasons for interest (POIs) proposals for social customers. In our approach, customer slant focuses, for instance, social, cityscapes, or noteworthy point, are removed from the geo-tag obliged artistic delineation of photos through the maker topic show instead of just from the geo-marks (GPS ranges). Central focuses and pervasive execution of our approach are appeared by expansive examinations on a broad gathering of data. Existing techniques generally encounter the evil impacts of various weaknesses. For example, sparsely can basically spoil the execution of customary CF. In case a customer just visits not a lot of zones, correct tantamount customer unmistakable evidence ends up being to a great degree testing on account of nonattendance of sufficient information for intense enlistment. Additionally, existing proposition approaches consistently dismiss rich customer information like printed delineations of photos which can mirror customers' travel slants. The point appears (TM) system is an effective way to deal with understand the "sparsely issue," yet is still far from elegant.

Zekeriya Erkin, Michael Beye, Thijs Veugen in 2012. Proposed methodology uses homomorphism encryption and secures multiparty estimation (MPC) techniques, which introduce a basic overhead in computational multifaceted nature. The second responsibility of this paper lies in minimizing this overhead by squeezing data. The progressions are spoken to by a multifaceted nature examination. Online recommender structures enable modified organization to customers. The major synergistic isolating frameworks take a shot at security tricky customer data, which could be manhandled in case it is spilled or by the master association himself. To guarantee customer's security, we propose to scramble the data and make recommendations by 
taking care of them under encryption. Thusly, the pro community watches neither customer slants nor recommendations.

Frank McSherry, Kunal Talwar in 2007. Keeps up a vital separation from the inconveniences of formally depicting exposures through a definition those strengths relative confines the change in the probability of any event. As far as possible the relative change in probability of events consequently of changes in any one customer's data. It is normal to expect that this general framework can be associated more exhaustively to other segment arrange issues. Not ready to keep up the hard assistant goals. Evaluating issues have the property that their outcome space is of low dimensionality, which prompts to noninsignificant base measure around the perfect game plan.

Geonhyeok Go, Joonhyuk Yang, Hyunwoo Park, Sangki Han in 2010. It should be adequately plentiful for exact customer slant showing and avoiding sparsely issue of unequivocal rating. It should have the ability to be acquired from unreservedly open space with clear mining framework, and without troublesome human effort and security encroachment. Utilize the direct of customers through electronic systems administration media as evident info, which does not require an additional cost except for an essential web mining. Likewise the recommender system using unequivocal data when in doubt encounters asserted sparsely issue. As needs be of the drawback in data, using unequivocal assessments as data set decays the way of proposition structures. Express assessments, in any case, are picked up by a monotonous method and require challenging human attempts to be made. In this way the recommender structure using express data as a rule encounters assumed sparsely issue.

Ziqi Wang, Yuwei Tan, Ming Zhang in 2010. Novel proposition figuring, which relies on upon casual associations. The interpersonal association is developed among customers and things, considering both the information of assessments and names. Calculation is support on the unpredictable stroll with start again yet makes open a more recognized and effective way to deal with address casual associations. Having considered the effect of marks, the move system is denser and the recommendation is more correct. The substance based proposal, which recommends resources in perspective of their substance and not on customer's evaluating and conclusion. The things are described by their related components of substance in the substance based structure. The disadvantage of this system is that the benefits ought to be essential and the embodiment of customers should be delineated in the parts of the substance.

Cem Tekin, Mihaela van der Schaar in 2013. To develop a novel online learning computation for decentralized Big Data portrayal using setting information about the high dimensional data. Shown sub coordinate mourn comes to fruition for this computation and demonstrated by method for numerical results that it beats gathering learning approaches in a framework security application. The issue of joint gathering by the appropriated and heterogeneous learners from various data sources as a flowed consistent scoundrel issue where each data is depicted by a specific setting. Electronic learning in scattered data course of action systems expects to address the edifying decentralization, correspondence costs and security issues developing in these structures.

Cem Tekin, Simpson Zhang, and Mihaela van der Schaar in 2014. Shown a novel course of action of estimations for multipro learning inside a decentralized casual association, and depicted the effect of different framework structures on execution. Novel game plan of computations can achieve sub linear regret in all cases, with the mourn being much humbler if the customer's affirmation probabilities for different things are free. By planning decentralized and using novel game plan of figuring's, administrators have the upside of holding their autonomy and insurance while so far fulfilling close perfect execution. Basic, complex mediator who holder basically gets to a parcel of rockets, and who simply get pointer about this subset, takes part to extend their total reward. Second, every master can pick different rockets at each calendar opening, which detail the arm gathering inconvenience combinatorial in the figure of arms. Figure this issue as a pleasing applicable pirate issue, legitimately bound the execution of the merchants diverged from the best proposition approach given the aggregate affirmation of customer sections and the supply of things, and likewise the setting subordinate purchase probabilities of everything, and check our results through numerical.

\section{PROPOSED SYSTEM}

Here we proposed the Quality of administration as well as increment the nature of the application. Here we dissecting the client's behavioral data from the every single client's action like inquiry recordings utilizing sub class and common pursuit. Here the clients characterized into sub classification in light of their interests. At the season of enlistment they will pick the classification of intrigue. Each client's have prescribed recordings in view of their advantage. Client's if has an opportunity to suggest random recordings clients can evade that utilizing not at all like alternative. Here we suggest the recordings in light of the clients look watchword premise. Every last recordings and sub classification seek in view of the video tag as it were. In the event that any of disconnected recordings suggested for you utilizing loathe alternative you can stay away from the video from your record. On the off chance that most extreme number of clients maintain a strategic distance from similar recordings that video consider as superfluous to this classification. Here something else is we can maintain a strategic distance from the superfluous substance promoter moreover. On the off chance that the greatest number of video kept away from similar supplier he will consider a substance promoter.

\section{CONCLUSION}

Our system recommends channels based on their viewing pattern and interests. The users just need to select the channel that they want to watch.In future research efforts, we will focus on those characteristics of upcoming intelligent devices such as iPhone, iPad etc.

\section{REFERENCES}

[1] Ming Cheung,James She, and Zhanming Jie, "Connection discovery using big data of user-shared images in social media," Multimedia, IEEE Transactions on.vol. 17, no. 9, pp. 1417-1428, 2015.

[2] Arjmand Samuel, Muhammad I. Sarfraz, Saleh Basalamah, Arif Ghafoor,"A framework for composition and enforcement of privacy-aware and context-driven authorization mechanism for multimedia big data," Multimedia, IEEE Transactions on. vol.17, no. 9, pp. 14841494, 2015.

[3] Marko Tkalcic, Andrej Košir, Jurij Tasic, "Affective labeling in a content-based recommender system for images," Multimedia, IEEE Transactions on. vol. 15, no. 2, pp. 391-400, 2013

[4] Shuhui Jiang, Xueming Qian, Jialie Shen, Yun Fu, Tao Mei, "Author topic model-based collaborative filtering for personalized POI recommendations," Multimedia, IEEE Transactions on. vol. 17, no. 6, pp. 907-918, 2015.

[5] Zekeriya Erkin, Michael Beye, Thijs Veugen, "Generating private recommendations efficiently using homomorphic encryption and data packing," in IEEE Transactions on 
Information Forensics and Security, vol. 7, no. 3, pp. 10531066, 2012.

[6] Frank McSherry, Kunal Talwar, "Mechanism design via differential privacy,"in Foundations Computer Science, FOCS07. 48th Annual IEEE Symposium on. IEEE, pp. 94103, 2007.

[7] Geonhyeok Go, Joonhyuk Yang, Hyunwoo Park, Sangki Han, "Using onlinemedia sharing behavior as implicit feedback for collaborative filtering," in Social Computing (SocialCom), 2010 IEEE Second International Conference on. IEEE, pp. 439-445, 2010.

[8] Ziqi Wang, Yuwei Tan, Ming Zhang, "Graph-based recommendation on social networks," Web Conference (APWEB), 2010 12th International Asia Pacific. IEEE, pp. 116-122. 2010.

[9] Cem Tekin, Mihaela van der Schaar, "Distributed online big data classification using context information," in Communication, Control, and Computing (Allerton), 51st Annual Allerton Conference on IEEE, pp. 1435-1442, 2013.

[10] Cem Tekin, Simpson Zhang, and Mihaela van der Schaar, "Distributed online learning is social recommender systems," in IEEE Journal of Selected Topics in Signal Processing, vol. 8, no. 4, pp. 638-652, 2014. 